\title{
Measurement of Maximum Deviation from Roundness Based on the Inverse Kinematics Principle
}

\author{
Tomáš Stejskal ${ }^{1}$, Miroslav Dovica ${ }^{2}$, Tatiana Kelemenováa ${ }^{2}$, Dominika Palaščáková ${ }^{1}$ \\ Eduard Jakubkovič ${ }^{2}$, Michal Považan ${ }^{1}$, Štefan Ondočko ${ }^{1}$ \\ ${ }^{1}$ Technical University of Košice, Faculty of Mechanical Engineering, Department of Production Engineering, Letná 9, 040 \\ 02 KošiceSlovakia,tomas.stejskal@tuke.sk \\ ${ }^{2}$ Technical University of Košice, Faculty of Mechanical Engineering, Department of Biomedical Engineering and \\ Measurement, Letná 9, 04002 Košice Slovakia.
}

\begin{abstract}
The article deals with a special method of measuring the maximum deviation of objects from roundness based on the inverse kinematics principle. The inverse measurement mechanism is based on the immobility of the measuring probes and the object performing all the motions required to measure a dimension. The advantage of this principle is minimization of the temperature change, while the adverse effect in the measurement system is greatly reduced at the same time. The measurement methodology requires a special software evaluation of the data measured. The aim of the given measurement methodology was to establish the maximum roundness deviation that corresponds to the Least Squares Circle (LSC) method. An experiment with three measuring probes was conducted to verify the methodology.
\end{abstract}

Keywords: Inverse kinematics, displacement sensor, roundness measurement.

\section{INTRODUCTION}

The measurement methodology focuses on measuring a single dimension of an object through inverse kinematics. According to this principle, the measuring motions with respect to the coordinate measuring system are performed by the object and the measuring system is stationary. This principle works best when proximity distance sensors are employed. The relative position of the sensors is fixed. In contrast, the object is a source of random values in the coordinate system. The randomness is caused by the object's motion and by the inaccuracy of its repeated arrival into the same positions relative to the sensor coordinate system. The nature of this measurement satisfies the Monte Carlo simulation principle. Therefore, the results of the evaluation are obtained by a statistical experiment, where local extremum is sought after.

A special measurement evaluation can estimate the degree of uncertainty in measuring an object's maximum deviation from roundness. Direct measurement of roundness along the perimeter of the object is only possible with the use of a circular positioning table, which is not the focus of the methodology. A more detailed explanation is provided in Chapter 3.

Known principles of measuring the roundness are based on rotation of the object against a fixed coordinate system of the measuring machine (for example, by Rouncomnex). This measuring system has been used in works by [1], [2]. In
CMMS, all the motions are performed by the system and the object is stationary (for example, in the Vast-multipoint measurement by Zeiss). The uncertainty of the measurement error arises from the measuring system's kinematic relations of motion. The issue of measurement uncertainty is discussed in [3], [4].

The new measurement principle is based on an unchanged position of the coordinate system, connected in the plane with three sensors. All other inaccuracies result from the random motion of the object in this space. Thus, the co-ordinate system does not take on kinematic kinetic relations. This feature is beneficial in terms of increasing the measurement uncertainty. On the other hand, the randomness and uncertainty of the object's position yield only statistical data, which is a disadvantage in terms of measurement uncertainty.

The inverse kinematics measurement method is similar to the modern method of measuring position prediction by multilateral precision based on the use of three laser trackers. The fundamental difference is that with multilateral measurement uncertainty, the angular motion is performed by the laser heads of the trackers and not by the object. The measured object dimension is evaluated by means of trigonometric formulas and the assessment of statistical uncertainty [5]. A similar method is also used to measure the geometric measurement uncertainty of an NC machine directly during change in the spindle position relative to the table [6]. 


\section{SUBJECT \& METHODS}

According to ISO 4291, the roundness deviation, when compared to ideal conditions, is understood to be the difference between the largest and the smallest radius of the measured component [7]. The result of the roundness deviation measurement depends mainly on the number and distribution of the measuring points and on the ideal element selected. The ISO 6318 standard prescribes for roundness 4 possible methods for selecting the ideal element: the LSC, MCC, MIC, and MZC [8], [9].

Deviation from roundness is most often checked through computer-assisted, special touch devices, where either the measuring contact (e.g., a CMM in case of the coordinate measuring technology) rotates around a fixed component or, inversely, the component rotates around the rotary axis and the deviations are continuously scanned by a dial indicator or other touch sensitive element working on a dial indicator principle (e.g., profilometers or contourographs). Measurement using these instruments is performed through continuous scanning of the curve on the workpiece surface and measuring the magnitude of deviation from the reference profile. When rotating a round-shaped part or rotating a measuring contact, any surface unevenness in a particular section is eventually identified. The number of points scanned during roundness measurement over the circumference of the entire component is usually high, therefore the measurement uncertainty is small.

For industrial purposes, it is advisable to measure roundness already during the process of component manufacturing. A special system is developed with a machine vision (camera), work holding tools, lighting device and also image processing software for roundness evaluation [10]-[12].

The advantage of contactless systems is, in particular, fast data acquisition (compared to touch methods) and also the fact that there is no contact with the workpiece, which might have otherwise resulted in its deformation and affect the measurement result by the scanning system. Compared to the conventional method of measuring roundness deviation (by roundness tester Roundtest RA-100), the experimentally built measurement system with a CCD camera and an image analysis software based on sophisticated algorithms showed a difference in measurement results worse by $7.22 \%$ [13], [14].

In case of coordinate measuring machines, the circle can be measured according to a user-defined number of selected points in addition to continuous curvature measurement. The measurement uncertainty of the roundness deviation in this case depends on the correct setting of the measurement parameters, in particular the number and distribution of the measuring points, the correct selection of the reference element, or the correct filtering of the data measured. The recommended number of measuring points for the selected ideal elements has been established experimentally [15]

Other contactless methods of roundness measurement use, e.g. capacitive displacement sensors [16] or laser sensors [17].

The simplest method is a two-point method, which is often used to check shafts. The two-point method utilizes the opposite measurement of the shaft diameter using a simple uniaxial measuring instrument, such as an outside micrometer or a caliper. The calculated mean values, the center position and the form deviation itself show large errors [14], [9].

\section{PRINCIPLE OF INVERSE KINEMATICS MEASUREMENT}

The circle in the plane is unambiguously defined by three parameters. As a rule, the parameters of the center of the circle and of its radius are provided. A case with such input information is suitable for classic roundness measurements, where the object rotates around an exactly defined center of rotation relative to the coordinate system. The measuring head is placed near the object's radius and is fitted to the measuring instrument frame.

What is checked in case of inverse kinematics is the possibility of using a modified measurement system. The object rotates in a plane, but the center of rotation is not precisely defined in relation to the coordinate system.

Theoretical calculation is based on perfect geometrical form.

In this configuration, the perfect circle is uniquely defined by three circumferential parameters with a fixed measurement angle and a distance from the object. By knowing the three values obtained from the object's perimeter, a calculation can be made about whether the three points lie on a circle of some diameter, or about the magnitude of their maximum deviation. By rotating the object in the plane, it is possible to measure another three values and then establish the diameter and the center of the new perfect circle.

Mathematical relations are based on plane geometry.

To measure a perfect circular object in the plane, 3 measurands are required. Conventional methods work with the center of rotation established in the plane (two values) plus the radius (third value). In our measurement arrangement, three deviations from the circumference of the circle are given in fixed measurement directions (Fig.1.). When the position is defined in this way, both the position and the size of the circle is unambiguously established. However, a special conversion of geometric dimensional data is required. The fixed coordinate system is formed by fixed sensor position.

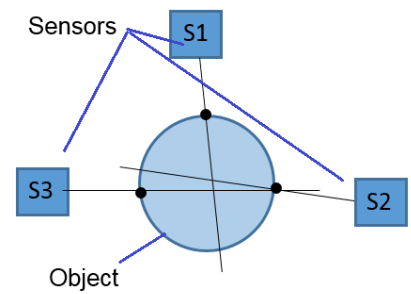

Fig.1. Principal arrangement of proximity sensors in the measurement plane.

The circle in the plane is clearly defined by three Cartesian coordinates. The scheme for calculating the circle's radius or diameter is based on goniometric formulas. For this purpose, a computational model was created, directly converting the measured data into a radius value. The data variances thus obtained satisfy the purpose of estimating the geometric 
component of the measurement error. The object has a floating center of rotation, which places a special requirement on how the dimension is calculated. Fig.2. presents a schematic diagram of the circular object's displacement. Values $\triangle A, \Delta B, \Delta C$ are measured by probes. The input data is defined by the initial values $A_{0}, B_{0}, C_{0}$. The output data is the circle's radius calculated from the measured data. A cosine formula in vector calculus is used for the conversion. The calculation scheme is verified by the program.

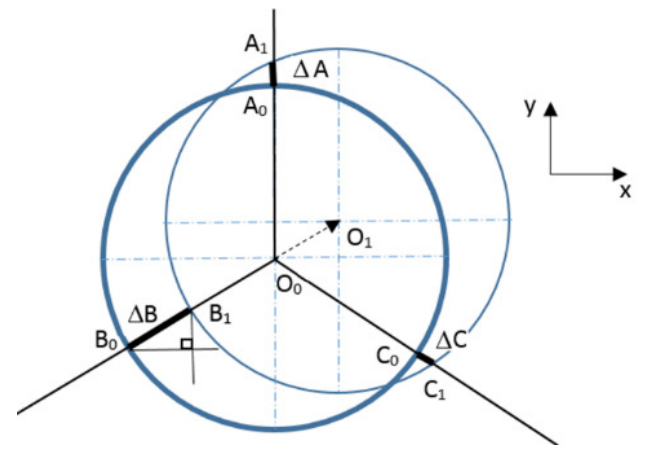

Fig.2. Scheme for calculating point displacement with respect to the coordinate system.

In order to use the calculation, it is necessary to know directions of the sensor axes with respect to the coordinate system selected. Based on this knowledge, components of displacement in rectangular directions can be calculated. For example

$$
B_{0}-B_{1}=\Delta B\left[B_{x}, B_{y}\right]
$$

The conversion formula used is based on the equation of the circle defined by three points in the plane (Fig.3.).

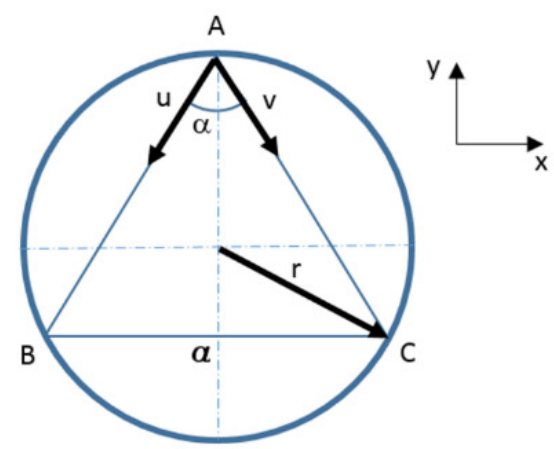

Fig.3. Scheme for calculating the radius of the circle from three points.

$$
r=\frac{\sqrt{\left(C_{x}-B_{x}\right)^{2}+\left(C_{y}-B_{y}\right)^{2}}}{2 \sin \left(\arccos \frac{u_{x} v_{x}+u_{y} v_{y}}{\sqrt{u_{x}^{2}+u_{y}^{2}} \sqrt{v_{x}^{2}+v_{y}^{2}}}\right)}
$$

Example of calculating position displacement of a single point in the $\mathrm{x}$-axis direction.

$$
B_{1 x}=B_{0 x}+\frac{\Delta B}{\sqrt{1+\left(\frac{B_{1 y}-B_{0 y}}{B_{1 x}-B_{0 x}}\right)^{2}}}
$$

Theoretical conversion relations derived from the schemes (Fig.2. and Fig.3.) demonstrate the applicability of the measurement method.

Thus, in theory, it is possible to distinguish which change in the triad of data is merely a displacement of a perfect circle's center and which represents the change in the diameter of the perfect circle, in addition thereto. The change in diameter indicates a deviation from roundness.

Geometric relations represent the internal relationships between the object of measurement and the sensors. By their nature, they contribute to the overall uncertainty of measurement, as there are no perfect objects and infinite precision in the real world. The Monte Carlo statistical method for evaluating the deviation from the reference condition rests on the knowledge of internal relations expressed by equations (2) and (3), respectively. It is exactly this that shall be utilized in the measurement methodology proposed.

\subsection{Theoretical treatise on inaccuracies in inverse measurement kinematics}

Fig.4. quantifies the effect of phenomena that increase the uncertainty of measuring the maximum deviation from roundness. The measurement is arranged by three sensors and an object moving in space with deviations from roundness unknown.

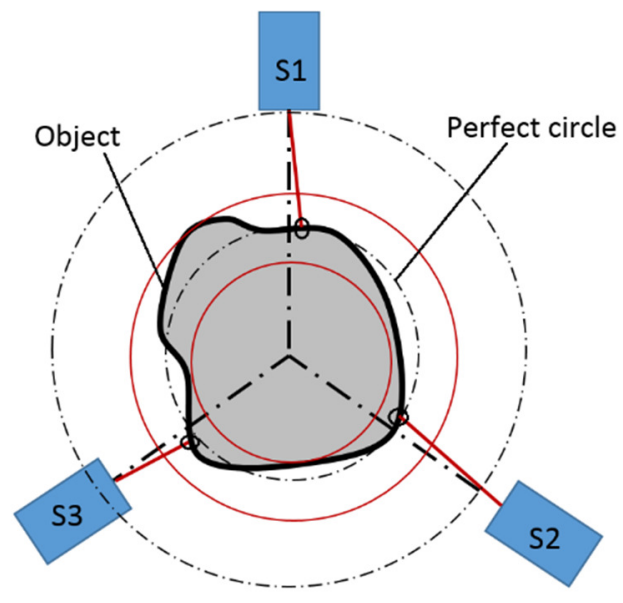

Fig.4. Uncertainty of measurement by three probes.

The following factors contribute to the measurement uncertainty:

Geometric factors:

- Contributions from the inaccurate geometry of the proximity probes' established direction

- Contributions from imperfect flatness on which the object rotates 
- Contribution from the variable position of the center of rotation, at which the distance between the object and the probe, and the associated nonlinearity of the probe, change

- Contribution from calculation error of geometric relations between the object and the sensors

Instrumentation factors:

- Sensor sampling rate and measurement density

- Accuracy of the measured value

- Distance sensor non-linearity

- Reflectivity of object surface

- Uncertainty of sensor measurement

- Repeatability

- Measuring point size

- Sensor response time

Dynamic aspects of measurement:

- Non-uniform of object rotation in space

- Vibration transmitted to the object and to the measurement assembly

Environmental effects:

- Temperature changes during measurement and the associated thermal expansion

- Change in ambient humidity

Contribution from object non-roundness

- Number of repeated measurements

- Frequency of occurrence of the measuring points along the perimeter

As you can see, the measurement is influenced by a very large number of factors, among which considerably complex and in many ways unexplored relationships exist. Therefore, the effectiveness of the retroactive process of calculating the average change based on geometric relationships is poor. The three measured values carry information on deviations from roundness, but direct calculation poses a problem.

It is, therefore, advisable to look for a parameter that sensitively responds to a change in dimension but, at the same time, is sensitive to displacement of the object's center of rotation.

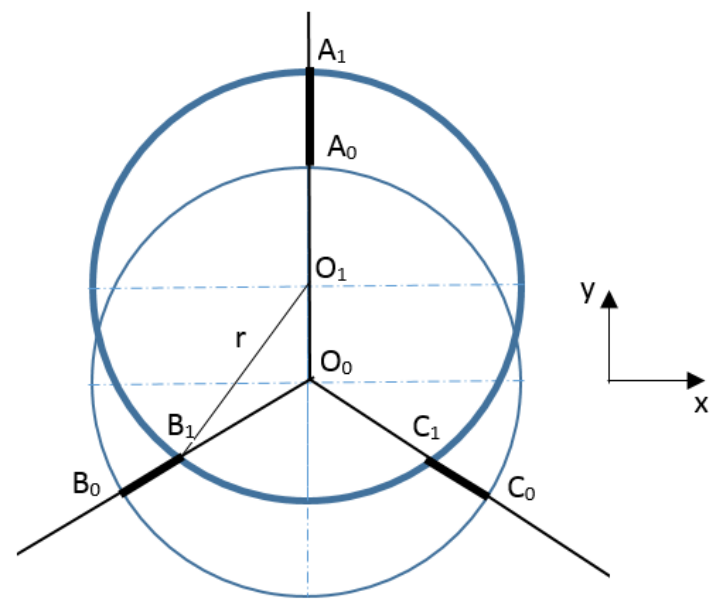

Fig.5. Scheme of calculating the displacement in the y axis direction.
To illustrate this, the following simple case of moving an object with respect to the coordinate system is shown. Let the object move only in the $y$-axis direction. The conversion relations (4) between the changes in values are as follows (Fig.5.):

$$
\Delta B=r-\frac{r \sin \left(\pi-\frac{2 \pi}{3}-\arcsin \frac{\sin \frac{2 \pi}{3} \Delta A}{r}\right)}{\sin \frac{2 \pi}{3}}
$$

The relation (4) is not linear and it also depends on the degree of measurement uncertainty to which the sensor is set in direction of the axis. However, it has a significant quality with regard to data triplet. A common parameter, suitable for statistical processing, is created for the triplet. As the common parameter, the quadratic mean of a value triplet was selected.

$$
W=\sqrt{(\Delta A)^{2}+(\Delta B)^{2}+(\Delta C)^{2}}
$$

Change in the common parameter depends on the extent of displacement from the reference position. As shown in Fig.8, this parameter increases with any deviation from the reference value. This fact will be used in statistical processing of measurement data. For statistical evaluation, comparisons with reference artifact measurements are used. From deviations measured on the examined object, the real deviation from the reference values is established.

From the amount of repeated measurements along the perimeter, a statistical set of the $W$ parameter is obtained. This set shows a variance of values due to many measurement uncertainties that have been listed above. However, the ratio of non-roundness to other effects is unknown.

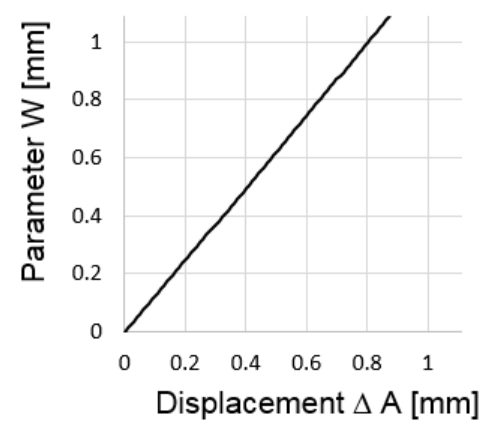

Fig.6. Dependence of the object displacement and the change parameter $W$.

In order to eliminate the effect of measurement uncertainty and to separate it from the effect of non-roundness, a reference measurement with an artifact must be performed. In this case, we know maximum deviation from roundness. Even with very precise measuring instruments, the measurement methodology cannot be more accurate than the 
uncertainty of the artifact shape itself. Therefore, when measuring an artifact, theoretically, we get the lowest variance of the $W$-values. Due to small differences in its values $(1 / 24$ of the object's diameter), the $W$ parameter's linear nature is acceptable (Fig.6.). Therefore, a linear relationship between the magnitude of the maximum deviation from roundness and the variance of the $W$ parameter can be used.

That is why a second reference measurement with a special artifact, where a large deviation from roundness is known, is necessary. Under unchanged measuring conditions, a greater variance of the $W$ parameter is obtained. In order to assign the measured variance a reference value of deviation from roundness, this difference must be statistically significant.

When measuring an unknown object, the $W$ parameter is

established under the same measurement conditions, and the ratio of the reference $W$ value and the measured $W_{R}$ value determines the maximum deviation from roundness $x_{v}$.

$$
x_{v}=\frac{W}{W_{R}} x_{v R}
$$

Where $W_{R}$ is the reference value obtained when measuring the artifact, $x_{v R}$ is the corresponding maximum deviation of the circularity of the artifact.

\subsection{Experiment preparation}

If the measurement is assessed from the statistical point of view, what is in fact available is a large number of data triplets $A_{n}, B_{n}, C_{n}$ which represent measured distances from the object's surface in the coordinate system. In theory, the first measurement is drawn upon, as it establishes the start of the coordinate system. All other position measurements show a deviation from the given reference position.

The basis for increasing the measurement uncertainty is a sufficiently large set of values measured on the measured object. Calculation between two overlapping effects, such as mechanism vibration and measuring instrument noise, is mathematically dealt with in [18] by correlation from 450 samples. 500 samples were also chosen for our experiment.

In order to establish the correct statistical deviation value, at least 30 measurements for one object position against the coordinate system are theoretically required, assuming a normal value distribution. The measurement uncertainty is established for the confidence interval 4 sigma.

The measurement methodology is based on the Monte Carlo method.

A selection of triplets of measured data that best characterize the measured dimension was used to establish the best uncertainty estimate and the mean deviation of the statistical set. Extreme statistical values result from the floating dynamics of the object's center of rotation and from the non-linearity of the static characteristics. To reduce measurement uncertainty, special software for measured data processing has been developed.

\section{MEASUREMENT PROCEDURE}

The measurement is done by rotating the object in the measuring space of the three triangulation sensors. 30 revolutions of the object were performed to obtain a sufficiently large statistical set.

The first measurement is a reference measurement. The artifact rotates in the measuring area. The dimension is most accurately characterized by the values obtained by the artifact. This measurement defines the degree of the methodology uncertainty.

Subsequent measurement is performed by the object under examination. There are two sets of triplet values that may point to a deviation from roundness. Only those data that are in the middle of the statistical variance are suitable for comparison. Outliers should therefore be excluded from the measurement, because they do not have statistical significance and increase measurement uncertainty. Outliers are most often the result of dynamic effects during measurement. These adverse effects can be reduced by a suitable rotary table with a motor drive.

\subsection{Selection of statistically significant values from the reference measurement of parmeter $W$}

For a file with only a single position measurement, statistical processing is a standard procedure. However, this method is indirect. The indirect status assessment methods were used in the work of [19]. A common parameter $W$ was used in our methodology. The evaluation draws on the values most frequently occurring during the reference measurement. Another requirement is that the deviations of the value measured and the value of the common parameter selected be minimal. Based on this principle, outliers at the distribution margins are excluded. In our case, the confidence interval was chosen $\pm 1 s$ from the distribution center, where $s$ is the sample standard deviation.

An example of the statistical distribution is shown in Fig.7. It is a time log of a parameter common for reference values. The confidence interval $2 s$ is indicated for the distribution.

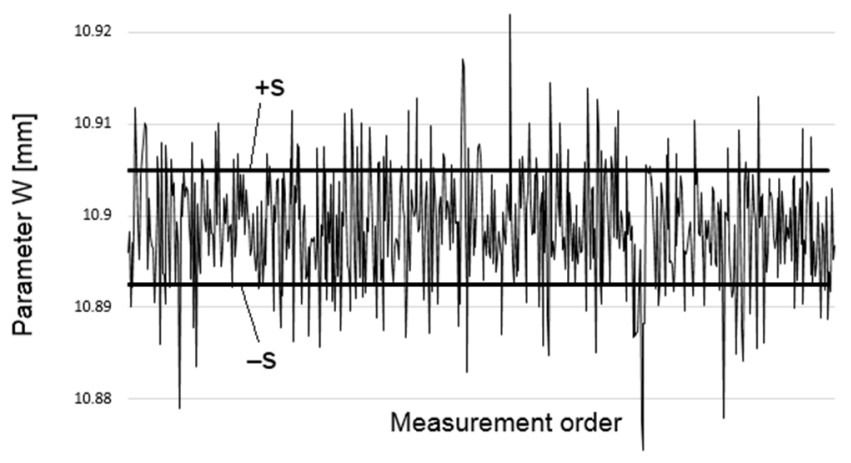

Fig.7. Log of the Common Reference Parameter $W$.

Values occurring most frequently have been used as reference values. The selection was made from the interval $2 s=0.0124 \mathrm{~mm}$. 


\subsection{Verifying the real artifact error on a $3 D M M S$}

The artifact was measured on the 3D measuring system Zeiss Contura. The coordinate measuring machine employed was equipped with a VAST XXT sensor which is a measuring sensor for scanning and single-point sensing. Maximum permissible error MPE for length measurement with Contura G2 (size 7/10/6) with RDS holder and VAST XXT sensor is $\mathrm{MPE}_{\mathrm{E}}=(1.8+L / 300) \mu \mathrm{m}$, where $L$ is measured length in $\mathrm{mm}$ (according to EN ISO 10360-2). Maximum permissible scanning probing error is $\mathrm{MPE}_{\mathrm{THP}}=3.5 \mu \mathrm{m}$ for required measuring time $68 \mathrm{~s}$ (according to EN STN ISO 10360-4) and maximum permissible error for form measuring is MPE $\mathrm{RON}_{\mathrm{N}}$ $=1.8 \mu \mathrm{m}$ (according to EN ISO 12181 and VDI/VDE 2617 part 2.2).

Fig.8. shows the results of the measurement protocol. The roundness deviation did not exceed the value $\Delta_{e}=0.002 \mathrm{~mm}$.

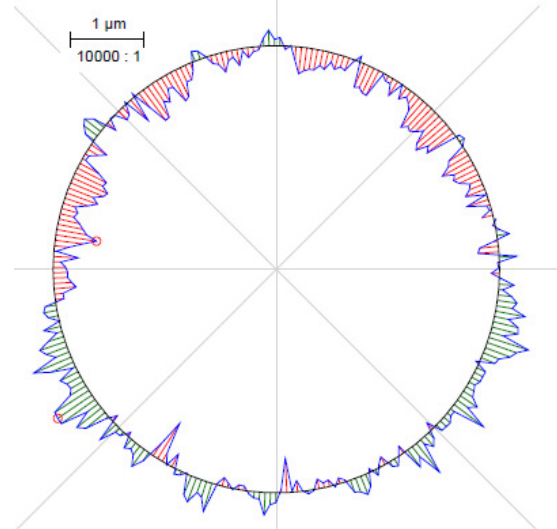

Fig.8. Log of the artifact's roundness on the 3D measuring system.

In the experiment the bearing artifact SKF 608-2Z SKF with cylinder tolerances IT6 was used.

Experiments were performed under laboratory conditions.

\subsection{Calculation of the measurement uncertainty of selected values}

A standard estimation procedure cannot be used to establish uncertainty because the measurement methodology is based on a comparison with an artifact whose size may not be known. The measurement results in establishing a total deviation that includes both a systematic and a random measurement component. But it does not define the dimension itself. The dimension is defined by the artifact value.

$$
x_{v R}=x_{e} \pm 2 u_{A}
$$

Where $x_{e}$ is the artifact dimension (in our case $x_{e}=24$ $\mathrm{mm}), u_{A}$ is a random component of the measurement error. Type A standard uncertainty was used to verify measurement uncertainty. The reason for this is a large number of statistical data, while the sources of random measurement errors are unknown.

$$
u_{A}=s_{x}=\sqrt{\frac{\sum_{i=1}^{n}\left(x_{i}-\bar{x}\right)^{2}}{n-1}}
$$

Where $S_{x}$ is the sample standard deviation and $\bar{x}$ is the average value of the measurements. Measurement uncertainty can also be evaluated in relation to the markers of process capability. The respective mathematical processing is the subject of [20]. The problem of estimating the measurement uncertainty in several independent distributions (populations) is addressed in the work [21]

\subsection{Algorithm for computing statistical deviation}

The algorithm comprises two nested cycles of comparisons of the values measured. Input reference values are made up of 500 measured triplets from different object positions. A common parameter $W$ was established and evaluated statistically. From a set of common parameter values, values that exceeded the limit of $\pm 1 s$ of the normal sample distribution were excluded by software. Values that fell within $\pm 1 s$ were used to evaluate the variance, or to assess the measurement uncertainty of the measurement method.

A common $W$ parameter was also established for all value triplets measured.

The first step of the cycle:

The least difference between the common artifact parameter and the common parameter of an individual measured triplet value is sought after.

Second step of the cycle:

After finding the minimum deviation from the common parameter, a deviation log is prepared for each sensor.

This two-step process is repeated until all measured values have been checked. The output is a log of minimum deviations from values of reference parameter $W_{R}$ by artifact measurment. Measurement uncertainty is established from the average difference of mean deviation values and so is the standard deviation for each sensor. For the purpose of measurement evaluation, a $4 \mathrm{~s}$ confidence interval for individual sensors was chosen (Table 1.).

Table 1. Result of deviation measurement in individual sensors.

\begin{tabular}{|l|c|c|c|}
\hline & \multicolumn{3}{|c|}{ sensor } \\
\cline { 2 - 4 } & $\mathbf{1}$ & $\mathbf{2}$ & $\mathbf{3}$ \\
\hline \hline $\begin{array}{l}\text { Diameter } r \text { middle } \\
\text { deviation [mm] }\end{array}$ & 0.0030 & -0.0073 & 0.0012 \\
\hline $\begin{array}{l}\text { Standard deviation } \\
2 s[\mathrm{~mm}]\end{array}$ & 0.0109 & 0.0216 & 0.0160 \\
\hline
\end{tabular}

The measurement uncertainty can be quantified by comparing the values measured on the object (artifact error) and the resulting inaccuracy under the methodology applied. Differences in deviations between individual sensors could also be attributed to their different distances from the object. 
The object is of a higher precision order than is the methodology of measurement. The object error is subtracted from the resulting deviation obtained by the given measurement methodology. The final formula proposed for calculating uncertainty is as follows:

$$
x_{v R}=x_{e} \pm\left(2 u_{A}-\frac{\Delta_{e}}{2}\right)
$$

Where the $u_{A}$ worst value from Table 1 is selected. In case of the experiment, the resulting measurement uncertainty is as follows:

$$
x_{v R}=24 \pm\left(2 \times 0.0216-\frac{0.002}{2}\right)=24 \pm 0.0422(\mathrm{~mm})
$$

This measurement uncertainty determines that roundness deviations smaller than the uncertainty range cannot be measured. In terms of standard measurements, the stated value is very large. However, the value depends on the type and accuracy of the sensor used.

\subsection{Verification of the method by simulated object error}

A deformed object was used for this purpose which, when measured, has to definitely show a greater deviation than is the uncertainty of measurement. The deviation is expressed by the maximum value of the quadratic mean parameter ( $W=0.9349 \mathrm{~mm})$, (Fig.9.).

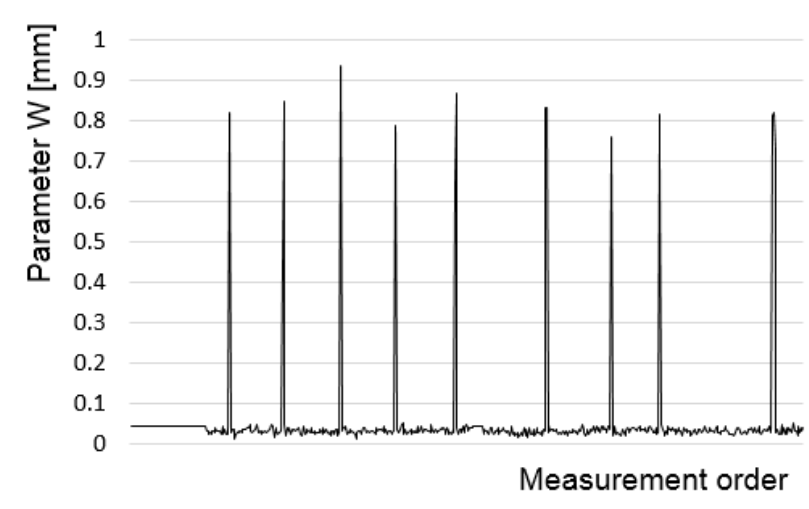

Fig.9. Parameter $W$ in simulated deviation from roundness.

\section{CONCLUSION}

The proposed measurement methodology verifies the applicability limits of the length measurement by inverse kinematics. The advantage of laser triangulation sensors is that they are contactless with a relatively large measuring range and good repeatability accuracy. High sensitivity of the triangulation sensor to change in measurement conditions when the systematic error of the sensor changes has proved to be a disadvantage, which prevents the method's full exploitation. However, the methodology itself brings certain benefits to be used in further development of the measuring systems.
Thermal compensation of the system can be performed shortly before the measurement of the unknown object by making reference measurements of the artifacts. Thermal changes will then only be bound to the net measurement time, which can be relatively short.

The second benefit is undemanding accuracy of the rotary table carrying the measured object. The methodology provides evaluation also in case of a floating center of rotation.

The disadvantage of the methodology is that any adjustment of the measurement conditions requires a new reference measurement of the artifacts. The measurement methodology can also be used to measure roundness at selected points. For this purpose, it is necessary to use an indexed rotary table and also adapt the evaluation of the $W$ parameter.

The paper deals primarily with the theoretical part of the methodology proposed. Its practical application can have a considerably wider range of use than shown in the validation experiment.

\section{ACKNOWLEDGMENT}

This contribution was supported by the Slovak Research and Development Agency (grants number APVV-15-0149 and APVV-18-0413) and by VEGA 1/0437/17, VEGA 1/0224/18, KEGA 025TUKE-4/2019, and KEGA 006 STU$4 / 2018$ grants.

\section{REFERENCES}

[1] El-Khabeery, M.M., Fattouh, M., El-Sheikh, M.N., Hamed, O.A. (1991). On the conventional simple spinning cylindrical aluminum cups. International Journal of Machine Tools and Manufacture, 31 (2), 203-219.

[2] Erenkov, O.Y., Zaev, V.V., Kartelev, D.V., Ashchin, V.A. (2009). Effect of priority deformation of polymer materials on surface layer quality for turned objects. Chemical and Petroleum Engineering, 45 (1-2), 105108.

[3] Raya, F.J., Cuesta, E., Martinez-Pellitero, S., Barreiro, J. (2015). Uncertainties on CMMs would be applied to the model of corrections based on Global Metric, Monte Carlo and Neural Network methods. Procedia Engineering, 132, 796-803.

[4] Swornowski, P.J. (2014). A new concept of continuous measurement and error correction in a coordinate measuring technique using a PC. Measurement 50, 99105.

[5] Aguado, S., Santolaria, J., Samper, D., Aguilar, J.J. (2016). Forecasting method in multilateration accuracy based on laser tracker measurement. Measurement Science and Technology, 28 (2), 025011.

[6] Deng, Y.F., Guo, J.J., Wang, J.D., Wang, H. (2012). The research and application of multi-point and timesharing measurement method on NC machine tool. In Materials Science Forum, 697-698, 249-252.

[7] International Organization of Standards. (1985). Methods for assessment of departure from roundness Measurement of variations in radius. ISO 4291. 
[8] International Organization of Standards. (1985). Measurement of roundness - Terms, definitions and parameters of roundness. ISO 6318.

[9] Sui, W., Zhang, D. (2012). Four methods for roundness evaluation. Physics Procedia, 24, 2159-2164.

[10] Ayub, M.A., Mohamed, A.B., Esa, A.H. (2014). In-line inspection of roundness using machine vision. Procedia Technology, 15, 807-816.

[11] Mohamed, A., Esa, A.H., Ayub, M.A. (2011). Noncontact approach to roundness measurement. In 2011 IEEE 7th International Colloquium on Signal Processing and its Applications. IEEE, 117-122.

[12] Vacharanukul, K., Mekid, S. (2005). In-process dimensional inspection sensors. Measurement, 38 (3), 204-218.

[13] Gadelmawla, E.S., Khalifa, W.M., Elewa, I.M. (2008). Measurement and inspection of roundness using computer vision. Mansoura Engineering Journal, 33 (3), M20-M32.

[14] Velasquez, J.D., Nof, S.Y. (2008). Integration of machine-vision inspection information for bestmatching distributed components and suppliers. Computers in Industry, 59 (1), 69-81.

[15] Gapinski, B., Grzelka, M., Rucki, M. (2006). The accuracy analysis of roundness measurement with coordinate measuring machines. In XVIII IMEKO World Congress: Metrology for a Sustainable Development.
[16] Xiuming, L., Zhaoyao, S. (2008). Application of convex hull in assessment of roundness error. International Journal of Machine Tools and Manufacture, 48 (6), 711-714.

[17] Mekid, S., Vacharanukul, K. (2011). In-process roundness measurement probe for turned workpieces. Measurement, 44 (4), 762-766.

[18] Pribil, J., Pribilova, A., Frollo, I. (2016). Comparison of mechanical vibration and acoustic noise in the open-air MRI. Applied Acoustics, 105, 13-23.

[19] Andris, P., Dermek, T., Frollo, I. (2015). Simplified matching and tuning experimental receive coils for lowfield NMR measurements. Measurement, 64, 29-33.

[20] Palencar, J., Palencar, R., Duris, S. Pavlasek, P. (2018). Correction of capability index computation based on uncertainty of the check standard in measurement process. Journal of Physics: Conference Series, 1065 (7), 072012

[21] Witkovsky, V., Wimmer, G., Duris, S. (2015). On statistical methods for common mean and reference confidence intervals in interlaboratory comparisons for temperature. International Journal of Thermophysics, 36 (8), 2150-2171.
Received June 5, 2019 Accepted October 31, 2019 